\title{
New Vaccine Introduction and Childhood Vaccination Timeliness in Two Urban, Informal Settlements in Nairobi, Kenya
}

\author{
Cara Bess Janusz, ${ }^{1,2 \star}$ Martin K. Mutua, ${ }^{3}$ Abram L. Wagner, ${ }^{1,2}$ and Matthew L. Boulton ${ }^{1,2,4}$ \\ ${ }^{1}$ Department of Epidemiology, School of Public Health, University of Michigan, Ann Arbor, Michigan; ${ }^{2}$ The Institute of Global Health Equity, \\ University of Michigan, Ann Arbor, Michigan; ${ }^{3}$ Data Measurements and Evaluation, African Population and Health Research Center, Nairobi, Kenya; \\ ${ }^{4}$ Department of Internal Medicine, Infectious Disease Division, Michigan Medicine, Ann Arbor, Michigan
}

\begin{abstract}
New vaccine introduction accompanied by social mobilization activities could contribute to improved routine immunization timeliness. This study assesses the impact of Kenya's introduction of pneumococcal conjugate vaccine (PCV) on the timeliness of routine childhood vaccination in two informal, urban settlements in Nairobi. Data collected from 2007 to 2015 as part of a demographic surveillance system were used to estimate annual vaccination delays of $\geq 4$ weeks among children aged 12-23 months in the period before and after the introduction of PCV in Kenya. Binomial segmented regression models using generalized estimating equations examined the association between vaccine introduction and timeliness of routine immunization. Over half of all children vaccinated in the two urban areas received one or more doses $\geq 4$ weeks after the recommended age. The timeliness of routine immunization showed slight improvements or nonsignificant changes during the years following PCV introduction compared with the preceding years (adjusted prevalence ratio [aPR]: $0.67,95 \% \mathrm{Cl}: 0.45-0.99$ for Bacille Calmette-Guerin receipt; aPR: 0.59, 95\% Cl: 0.41-0.83 for third dose Pentavalent receipt; aPR: 1.19, 95\% Cl: 0.99-1.42 for measles). However, as of 2015, delayed vaccination remained prevalent in children, particularly among the poorest residing in the settlements. Many sub-Saharan African countries have introduced new life-saving vaccines into their routine childhood immunization schedule. Additional evidence regarding the positive or neutral influence of new vaccine introduction on the performance of delivery systems provides further justification to sustain the inclusion of these more costly vaccines in the immunization schedule.
\end{abstract}

\section{INTRODUCTION}

The development and broad adoption of new childhood vaccines has resulted in remarkable improvements in child health over the past two decades. ${ }^{1}$ However, at the time of their introduction into the global market, new vaccines were prohibitively expensive for low- and middle-income countries (LMICs), resulting in substantial lags in their adoption compared with in wealthier countries. ${ }^{2}$ Beyond the cost of vaccine supply, introducing new vaccines implies substantial financial burdens on ministries of health, such as upgrading outdated or strained immunization program infrastructure, improving surveillance systems, and investing in training and planning for effective delivery strategies. ${ }^{3}$ Despite these considerable financial and logistical obstacles, the lag in implementing new vaccine offerings has diminished in recent years for many parts of sub-Saharan Africa. ${ }^{4}$

The principle priority of contemporary immunization programs is achieving and maintaining high levels of uptake for all childhood vaccines in routine use, many of which are critical to disease control and elimination efforts. ${ }^{5}$ Effective protection against these diseases requires uniformly high levels of coverage in target populations at recommended age intervals, achieving "on-time" vaccination to protect children against disease at ages when they are the most susceptible to infection. ${ }^{6}$ That is, vaccines delivered too early may not confer adequate or long-lasting protection, and vaccines delivered too late may result in unnecessarily prolonged periods for which children are at risk of more serious illness or death from vaccine-preventable diseases. ${ }^{7,8}$ With the inclusion of new vaccines into routine programs, immunization schedules have become more complex and vaccination programs potentially

* Address correspondence to Cara Bess Janusz, School of Public Health, University of Michigan, 1415 Washington Heights, Ann Arbor, MI 48109. E-mail: cjanusz@umich.edu more strained. Although specific to only a few settings, qualitative research on the health systems impact of new vaccine introduction has suggested that existing challenges to chronically underfunded health systems and programs may be exacerbated, such as overextending the limited number of healthcare workers with new vaccine promotion activities and as a result unintentionally detracting from other high-priority disease elimination goals through immunization activities. ${ }^{3,9}$ By contrast, other studies have found that the adoption of new vaccines was perceived as an opportunity to retrain and strengthen healthcare workforce capacity and to further promote the benefit of immunization services as a whole, leveraging the availability of additional government resources or donor funding. ${ }^{10-12}$ Further, social mobilization, outreach, and information campaigns that often accompany the launch of new vaccines may promote higher uptake and timeliness of other vaccines recommended for routine use in communities, specifically because new vaccines are recommended for concomitant administration with other traditional vaccines at established age intervals for vaccination programs. ${ }^{13}$

Kenya has made immunizations a health priority, introducing new vaccines ahead of many other LMICs in subSaharan Africa, such as pneumococcal conjugate vaccine (PCV) in 2011 and rotavirus vaccine (RV) in $2014 .^{14}$ The Kenyan Expanded Program on Immunization was established in 1980 by the Ministry of Health to provide vaccinations and monitor vaccine-preventable childhood illness and death. As of 2020, Kenya's routine childhood immunization schedule includes one dose each of Bacille Calmette-Guerin (BCG) and oral polio vaccine (OPV) at birth; three doses each of diphtheria-tetanus-whole cell pertussis-Haemophilus influenzae type b-hepatitis B (Pentavalent[Penta]), OPV, and PCV at 6,10 , and 14 weeks; two doses of RV at 6 and 10 weeks; one dose of Inactivated Polio Vaccine (IPV) at 14 weeks; and two doses of measles and rubella at 9 and 18 months of age. ${ }^{15}$ To date, few studies have quantitatively assessed the influence of 
new vaccine introduction on immunization program outcomes. Of those published, ${ }^{16-20}$ none is specific to Kenya's experience with recent new vaccine introduction in urban poor contexts. In this study, we describe and explore the hypothesized beneficial impact of new vaccine introduction, using the experience of PCV as an example, on the timing of other vaccines recommended for routine use in two urban poor communities in Nairobi, Kenya. Using data from the Nairobi Urban Health and Demographic Surveillance System, we cross-sectionally assess annual trends in vaccination delays derived from the publicly available data collected on administration of routine immunization doses recommended before 12 months of age among children residing in the surveillance areas located in Viwandani and Korogocho, urban informal settlement communities within Nairobi, before and after the introduction of PCV.

\section{MATERIALS AND METHODS}

Study setting: The Nairobi Urban Health and Demographic Surveillance System. The Nairobi Urban Health and Demographic Surveillance System (NUHDSS) was established to study the health effects of migration and poverty in the urban capital of Kenya. Managed by the African Population and Health Research Center, the NUHDSS monitors health and demographic trends through the registration of all births, migratory movements, and deaths in two informal settlement communities, Korogocho and Viwandani. These two settlements were purposefully selected for prospective study following a cross-sectional assessment in 2000 of Nairobi informal settlements that found residents in these areas had worse health outcomes than any other settings in Kenya. ${ }^{21}$

Circular migratory patterns between rural Kenya and Nairobi are common for residents in both surveillance area settings, although the population in Korogocho has become more stable over time. Households in Korogocho are generally characterized by spousal co-residence and multi-generational families that relocated from rural areas of Kenya beginning in the 1960s. In contrast, Viwandani's proximity to neighboring industrial areas attracts younger household heads, often settling temporarily without their entire family units, and fewer than 5\% of residents are born in the settlement. ${ }^{22,23}$ Violence coupled with absent security measures, poor or lacking infrastructure for sanitation, and governance issues related to land ownership disputes pose serious challenges to improving the health and well-being of the populations residing in both communities. ${ }^{21,24}$ The African Population and Health Research Center and partner agencies have used data from the NUHDSS to inform policy and programmatic changes that aim to improve the living conditions and health situation in Viwandani, Korogocho, and other slums of Nairobi. ${ }^{25}$

All household units occupying dwellings in Korogocho and Viwandani were recruited for participation beginning in 2002. Since then, field interviewers visit the surveillance catchment every 120 days to update the register of participating residents, removing individuals who have out-migrated or died and incorporating births and new residents who have inmigrated. Demographic and migration pattern data are collected at recruitment for each member of the household. During subsequent field interview visits, information is collected on in- and out-migration, births, deaths, maternal and child health, and economic and food security for all registered households. ${ }^{21,23}$ As of 2018 , the NUHDSS monitored 88,974 individuals who reside in 33,462 households in Viwandani and Korogocho, and every year approximately 1,500 to 1,900 new live births are registered in the surveillance system. ${ }^{26}$

Study population sample: Children 12-23 months followed in the NUHDSS. As part of the maternal and child health focus of the system, the NUHDSS collects a record of vaccination for all children under 5 years of age who reside in the surveillance catchment. Mothers or caretakers of children under 5 years of age provide a complete history of vaccination, and interviewers review card documentation to verify the date and number of doses for each vaccine received. Vaccination status is updated in the surveillance system for each child at all subsequent visits until the schedule is complete or the child is no longer age eligible for this component of the survey. Data on children aged 12-23 months at the time of interview were used for the 4 years before and after the initial year of new vaccine introduction (2007-2015), identifying PCV as the first new vaccine to be incorporated into the schedule during the study period in 2011 . To reduce selection bias that may result from loss to follow-up or survivorship during the 12- to 23month period, the first vaccination record created in the system following 12 months of age was included for analysis. Vaccination records were matched to demographic data collected for children and their households in the same year or as close to the vaccination record year as possible. Children who never had a vaccination card reviewed or who did not have a card available in the 12- to 23 -month period were excluded from analysis.

Variables. The primary outcome was a binary variable of delayed versus on-time vaccination relative to the agespecific recommendations for vaccination at birth, 6 weeks, 10 weeks, 14 weeks, and 9 months of age in Kenya for all vaccines recommended prior to the introduction of new vaccines. Data for receipt of vaccine doses recommended at ages older than 12 months (i.e., second dose of measles at 18 months) were not collected during the study period. Delays were defined as any vaccine dose that was administered $\geq 4$ weeks after the recommended age of vaccination. All vaccines administered within the time interval between the recommended age and the 4-week delay window were considered acceptable timing for receipt and defined as "on-time." Vaccination records that reported age at vaccination before the child's birthdate or other implausible vaccination dates (e.g., measles vaccination at birth) were excluded from analysis. Vaccination status by 12 months of age, defined as receipt of all recommended vaccines by the age of 12 months, and dose-specific completion status variables were also derived for descriptive analysis. Data on the child's sex, age, ethnicity, and household wealth status were directly obtained from residency records. There was incomplete information in the system to identify all motherchild pairs, and therefore mother characteristics were not examined in this analysis.

Analysis. The distribution of demographic characteristics and vaccination status of children was explored over time, distinguishing between the before and after PCV introduction periods. The proportion of children who were delayed in their vaccination schedules was examined descriptively across three periods: 1) prior to PCV introduction (2007-2010), 2) during the year in which PCV was introduced (2011), and 3) the 
period following PCV introduction (2012-2015). To explore the influence of new vaccine introduction on the dose-specific timeliness of routine immunization in children $<12$ months, segmented binomial regression using generalized estimating equations was used comparing the pre-introduction period (2007-2010) with the post-introduction period (2012-2015). To maintain balance in the period comparisons, only data from 4 years before and after the introduction of PCV were used in the models. Acknowledging the limited data availability to control for potential time-dependent confounders, we only sought to explore the association in terms of a timedependent level change as opposed to changes in the trend trajectory, or slope, defining the estimated prevalence of delayed vaccination receipt over time. Prevalence ratios reflecting pre- and postintroduction period level changes with robust standard errors were calculated, accounting for correlation due to approximately $30 \%$ of children residing in the same household unit as other families with young children in the sample. Models included a term to control for annual trends in the outcome, given changes to the annual ageeligible catchment sample. Individual level factors that were hypothesized to influence the timeliness of vaccine receipt as well as the impact of new vaccine introduction on vaccination behavior were used to adjust models, including household wealth status, ethnicity, and study setting. All analyses were conducted using Stata V16.1 in December of 2020.

\section{RESULTS}

From January 2007 to December 2015, the NUHDSS registered 7,684 children 1 year of age living in Viwandani and Korogocho; 148 children were excluded from this study due to missing household or individual characteristic data. In total, $70 \%$ of these children had a vaccination card available for review at one or more interviews during the study period (Figure 1). The distribution of characteristics based on the primary inclusion criteria of having a vaccination card was relatively well balanced between children eligible for inclusion $(N=5,341)$ and those who were not $(N=2,195)$ for the 2007-2015 surveillance period. However, the proportion of children who had a card available at the time of interview varied year to year (minimum: $44 \%$ in 2010; maximum: $83 \%$ in 2012), and children with the availability of vaccination cards were members of marginally wealthier households. Children with cards were predominantly members of the Kikuyu ethnic group (26\%), the largest ethnic majority in all of Kenya, followed by other ethnicities (Luhya, 19\%; Luo, 20\%; Kamba, 19\%; Kisii, 6\%; others, 11\%). Among the children with vaccination cards, there was equal representation between the two surveillance sites and across sex (Table 1).

Demographic factors did not vary substantially across the periods before, during, and after PCV introduction into Kenya's routine immunization program. Overall, $36 \%$ of the

30,549 children 0.59 registered with 70,413 surveillance visits

Excluded 54,525 visits with children who

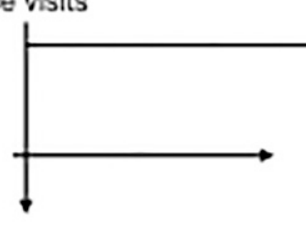
wete not 12.23 months at time of interview

Excluded 4,547 visits in children who did not ever have a vaccination history recorded in the 12.23 months period

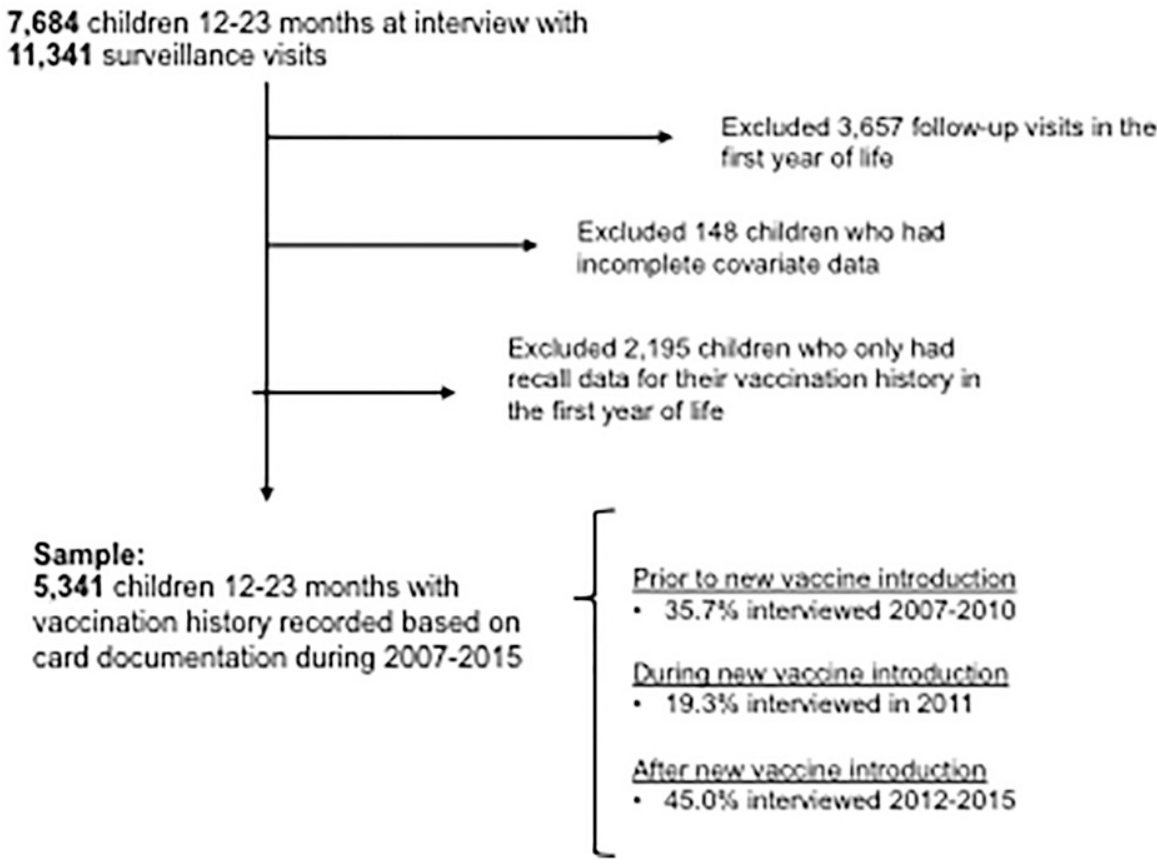

FIGURE 1. Study sample diagram of children 12-23 months registered in the Nairobi Urban Health and Demographic Surveillance System from 2007 to 2015 with vaccination data. 
TABLE 1

Characteristics of children 12-23 months identified in the Nairobi Urban Health and Demographic Surveillance System (2003-2015) according to the availability of a vaccination card

\begin{tabular}{|c|c|c|c|}
\hline & Overall & Card & Recall \\
\hline & & Frequency (\%) & \\
\hline Total & $7,536(100)$ & $5,341(100)$ & $2,195(100)$ \\
\hline \multicolumn{4}{|l|}{ Surveillance site } \\
\hline Korogocho & $3,570(47)$ & 2,616 (49) & $954(43)$ \\
\hline Viwandani & 3,966 (53) & $2,725(51)$ & $1,241(57)$ \\
\hline \multicolumn{4}{|l|}{ Sex } \\
\hline Male & $3,837(51)$ & $2,719(51)$ & $1,118(51)$ \\
\hline Female & $3,699(49)$ & $2,622(49)$ & $1,077(49)$ \\
\hline \multicolumn{4}{|l|}{ Ethnicity } \\
\hline Kikuyu & $1,948(26)$ & $1,402(26)$ & $546(25)$ \\
\hline Luhya & $1,469(19)$ & $1,068(20)$ & 401 (18) \\
\hline Luo & $1,278(17)$ & $908(17)$ & $370(17)$ \\
\hline Kamba & $1,591(21)$ & $1,119(21)$ & $427(22)$ \\
\hline Other & $1,250(17)$ & 844 (16) & 406 (18) \\
\hline \multicolumn{4}{|l|}{ Wealth quintile } \\
\hline Poorest & $1,043(14)$ & $665(12)$ & $378(17)$ \\
\hline Poor & $1,304(17)$ & $886(17)$ & $418(19)$ \\
\hline Middle & 1,455 (19) & $1,022(19)$ & $433(20)$ \\
\hline Rich & $1,810(24)$ & $1,281(24)$ & $529(24)$ \\
\hline Richest & 1,924 (26) & 1,487 (28) & $437(20)$ \\
\hline \multicolumn{4}{|l|}{ Year } \\
\hline 2007 & $816(11)$ & $517(10)$ & $299(14)$ \\
\hline 2008 & $1,114(15)$ & $865(16)$ & $249(11)$ \\
\hline 2009 & $667(9)$ & 307 (6) & $360(16)$ \\
\hline 2010 & $492(7)$ & $216(4)$ & 276 (13) \\
\hline 2011 & $1,276(17)$ & 1,031 (19) & $245(11)$ \\
\hline 2012 & 1,525 (12) & $1,264(24)$ & 261 (12) \\
\hline 2013 & 909 (12) & 721 (13) & $188(9)$ \\
\hline 2014 & $511(7)$ & $294(6)$ & $217(10)$ \\
\hline 2015 & $226(3)$ & $126(2)$ & $100(5)$ \\
\hline
\end{tabular}

sample was observed in the pre-introduction period $(N=$ $1,905), 19 \%$ was observed in the year of PCV introduction $(N=$ $1,031)$, and $45 \%$ was observed in the post-introduction period $(N=2,405)$. The average age of the child at the time of interview was 15-16 months across the three time periods. Greater than one-quarter of children were members of the richest households in the two communities over the same periods. In the two communities, the coverage of several basic vaccines in the schedule based on documented history or maternal recall at time of interview was consistently high over time: BCG > 98\%, Penta1 > 97\%, Penta2 > 94\%, and Penta3 > 90\%. However, the proportion of children who received OPV and measles varied across the three time periods, and fewer than three-quarters of children were reported as having received the recommended basic immunization schedule by 12 months of age for the periods after 2010 (Table 2).

Among children who had date verification of vaccination on their immunization cards, many were vaccinated $>4$ weeks after the recommended age. From 2007 to 2015, delayed vaccination as a proportion of all children vaccinated during the period was $13.8 \%$ for BCG recommended at birth; $18.2 \%$ and $20.8 \%$ for Penta3 and OPV3, respectively, recommended at 14 weeks; and $54 \%$ for measles vaccine recommended at 9 months (Table 3). Except for the persistently high and occasional increases in prevalence of delayed measles vaccination from year to year, the overall proportion of delayed vaccination across the schedule declined from 2007 to 2015 (Figure 2). Delayed vaccination was slightly more common in Korogocho, where, for example, late receipt of the final doses in the Penta and OPV series was substantially higher as
TABLE 2

Demographic factors and vaccination status among children 12-23 months registered in the Nairobi Urban Health and Demographic Surveillance System with a vaccination card by time period

\begin{tabular}{|c|c|c|c|}
\hline & $\begin{array}{l}\text { Period prior to new } \\
\text { vaccine intro } \\
(2007-2010)\end{array}$ & $\begin{array}{l}\text { Period during new } \\
\text { vaccine intro } \\
(2011)\end{array}$ & $\begin{array}{l}\text { Period after new } \\
\text { vaccine intro } \\
(2012-2015)\end{array}$ \\
\hline & $N=1,905$ & $N=1,031$ & $N=2,405$ \\
\hline $\begin{array}{c}\text { Mean age, } \\
\text { months }\end{array}$ & 15.72 & 16.40 & 15.13 \\
\hline \multicolumn{4}{|l|}{ Study site } \\
\hline Korogocho & $46.3 \%$ & $52.3 \%$ & $49.7 \%$ \\
\hline Viwandani & $53.7 \%$ & $47.7 \%$ & $50.3 \%$ \\
\hline \multicolumn{4}{|l|}{ Sex } \\
\hline Male & $49.8 \%$ & $51.7 \%$ & $51.4 \%$ \\
\hline Female & $50.2 \%$ & $48.3 \%$ & $48.6 \%$ \\
\hline \multicolumn{4}{|l|}{ Ethnicity } \\
\hline Kikuyu & $27.2 \%$ & $28.0 \%$ & $24.7 \%$ \\
\hline Luhya & $20.1 \%$ & $18.0 \%$ & $20.8 \%$ \\
\hline Luo & $17.6 \%$ & $17.6 \%$ & $16.3 \%$ \\
\hline Kamba & $21.4 \%$ & $21.0 \%$ & $20.6 \%$ \\
\hline Kissii & $6.1 \%$ & $4.8 \%$ & $6.5 \%$ \\
\hline Other & $7.7 \%$ & $10.7 \%$ & $11.1 \%$ \\
\hline \multicolumn{4}{|l|}{ Wealth quintile } \\
\hline Poorest & $14.0 \%$ & $11.1 \%$ & $11.8 \%$ \\
\hline Poor & $16.6 \%$ & $18.4 \%$ & $15.8 \%$ \\
\hline Middle & $18.4 \%$ & $20.1 \%$ & $19.3 \%$ \\
\hline Rich & $23.3 \%$ & $21.3 \%$ & $25.7 \%$ \\
\hline Richest & $27.6 \%$ & $29.1 \%$ & $27.5 \%$ \\
\hline \multicolumn{4}{|l|}{$\begin{array}{l}\text { Vaccines } \\
\text { received* }\end{array}$} \\
\hline BCG & $99.6 \%$ & $98.6 \%$ & $97.3 \%$ \\
\hline Penta1 & $98.5 \%$ & $97.8 \%$ & $97.0 \%$ \\
\hline Penta2 & $97.4 \%$ & $95.2 \%$ & $94.1 \%$ \\
\hline Penta3 & $94.4 \%$ & $89.9 \%$ & $90.7 \%$ \\
\hline OPV1 & $98.3 \%$ & $96.9 \%$ & $96.9 \%$ \\
\hline OPV2 & $96.8 \%$ & $93.3 \%$ & $93.9 \%$ \\
\hline OPV3 & $94.3 \%$ & $87.1 \%$ & $90.9 \%$ \\
\hline Measles & $85.9 \%$ & $78.0 \%$ & $73.8 \%$ \\
\hline $\begin{array}{l}\text { All vaccines } \\
\text { (by time of } \\
\text { interview) }\end{array}$ & $81.3 \%$ & $70.8 \%$ & $68.7 \%$ \\
\hline
\end{tabular}

compared with Viwandani (25\% versus $12 \%$ for Penta3 and $29 \%$ versus $13 \%$ for OPV3). Children whose families selfreported as Luhya or Luo ethnicities had higher levels of delays than the largest ethnic group (Kikuyu) for vaccines administered at birth and at 6,10 , and 14 weeks of age. The distribution of delays skewed much higher in the poorest households compared with the richest ones across all vaccination encounters (Table 3). Whereas the prevalence of delays in receipt of BCG, Penta1-3, and OPV1-3 varied according to several characteristics, delays in receipt of measles vaccine at 9 months occurred in more than $50 \%$ of children consistently across sociodemographic strata (Table 3).

Comparing vaccination by week of age between the preand post-introduction periods for PCV, a significantly higher proportion of children were vaccinated closer to the age of recommendation for BCG (birth), Penta1 (6 weeks), Penta3 (14 weeks), and measles first dose (9 months or 36 weeks) during the 2012-2015 period (log rank tests for each inverse Kaplan Meier vaccination curve: $P<0.001$ ) (Figure 3). Further, controlling only for calendar year of the interview, there was a statistically significant lower prevalence of delayed vaccination observed in the sample, comparing delays in the postintroduction period (2012-2015) with the preintroduction period (2007-2010) for doses administered at birth, 6 weeks, 10 weeks, and 14 weeks (results not shown). Delays in 
TABLE 3

Prevalence of delayed vaccination in children 12-23 months registered in the Nairobi Urban Health and Demographic Surveillance System before, during, and after PCV introduction according to demographic factors

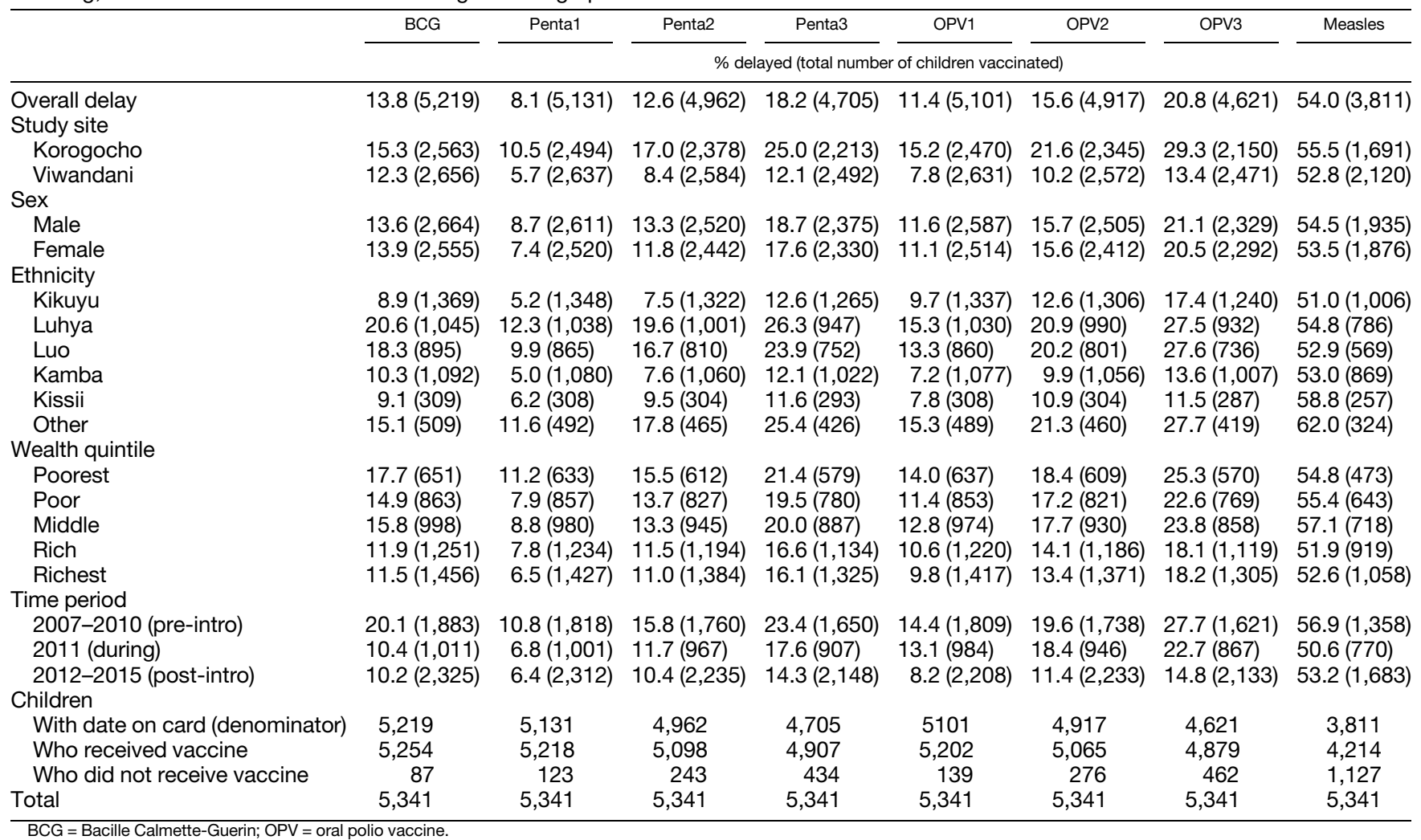

measles receipt showed a significant level change in the prevalence of delay in the years following the introduction of PCV compared with the period during pre-introduction (prevalence ratio [PR]: 0.93; 95\% Cl: 0.87-0.99). After adjusting for surveillance area, ethnicity, and wealth, the PR of vaccination delay in the post-introduction era compared with the pre-introduction era was slightly attenuated for BCG, Penta1, and Penta3, though the magnitude of association $<1$ continued to suggest lower prevalence of delays in the post-introduction period for these vaccines (Table 4). After adjusting for surveillance area, ethnicity, and wealth, the ratio in timeliness of measles vaccination between the two periods was not statistically significant (PR: 1.19; 95\% Cl: 0.99-1.42).

\section{DISCUSSION}

The introduction of PCV, as well as other childhood vaccines such as RV, and more recently one dose of inactivated poliovirus vaccine (IPV) and a second dose of measles, are important markers of progress for child health in Kenya. However, adding new antigens and additional doses to the childhood immunization schedule also imposes substantial financial and programmatic challenges for countries that have yet to achieve wide-reaching, timely access to the basic immunization schedule. Incorporating PCV in 2011 was the first of several revisions to the childhood immunization schedule in Kenya. Within 2 years of its adoption, coverage of the third dose of PCV had reached $>80 \%$ in children at the national level, thanks in part to a massive national catch-up program. ${ }^{27}$
In this study, using a quantitative approach, we found that new vaccine introduction was not associated with any significant decreases in the timeliness of other routine immunization services in two urban, poor communities in Nairobi. Evidencing a lack of disruption or deterioration of other routine immunization services in the presence of new vaccine introduction is paramount in settings that must balance the desire to innovate for child health with the need for continued investment in improving immunization coverage and timeliness overall.

Although our comparative assessment of routine immunization timeliness between the periods before and after PCV introduction was intended to serve as illustrative exploration of the influence of new vaccine introduction generally, our findings may contribute to the case for sustaining PCV in the routine schedule going forward. Kenya, like many LMICs, introduced new vaccines into the routine schedule with the support of co-financing from Gavi, the Vaccine Alliance, a public-private partnership created to improve access to new and under-used vaccines in the world's poorest countries. ${ }^{2}$ Gavi's funding agreements are designed to be time bound with a view to "graduate" country immunization programs from donor support and transition them to a fully self-financed model, using domestic resources to pay for the purchase and delivery of new vaccines. Countries subscribing to this financial model assume the full cost of new vaccines after the 3-year average gross national income per capita exceeds US $\$ 1,580 .{ }^{28}$ Using gross national income per capita projections for the current decade, Kenya is slated to complete its transition and become 


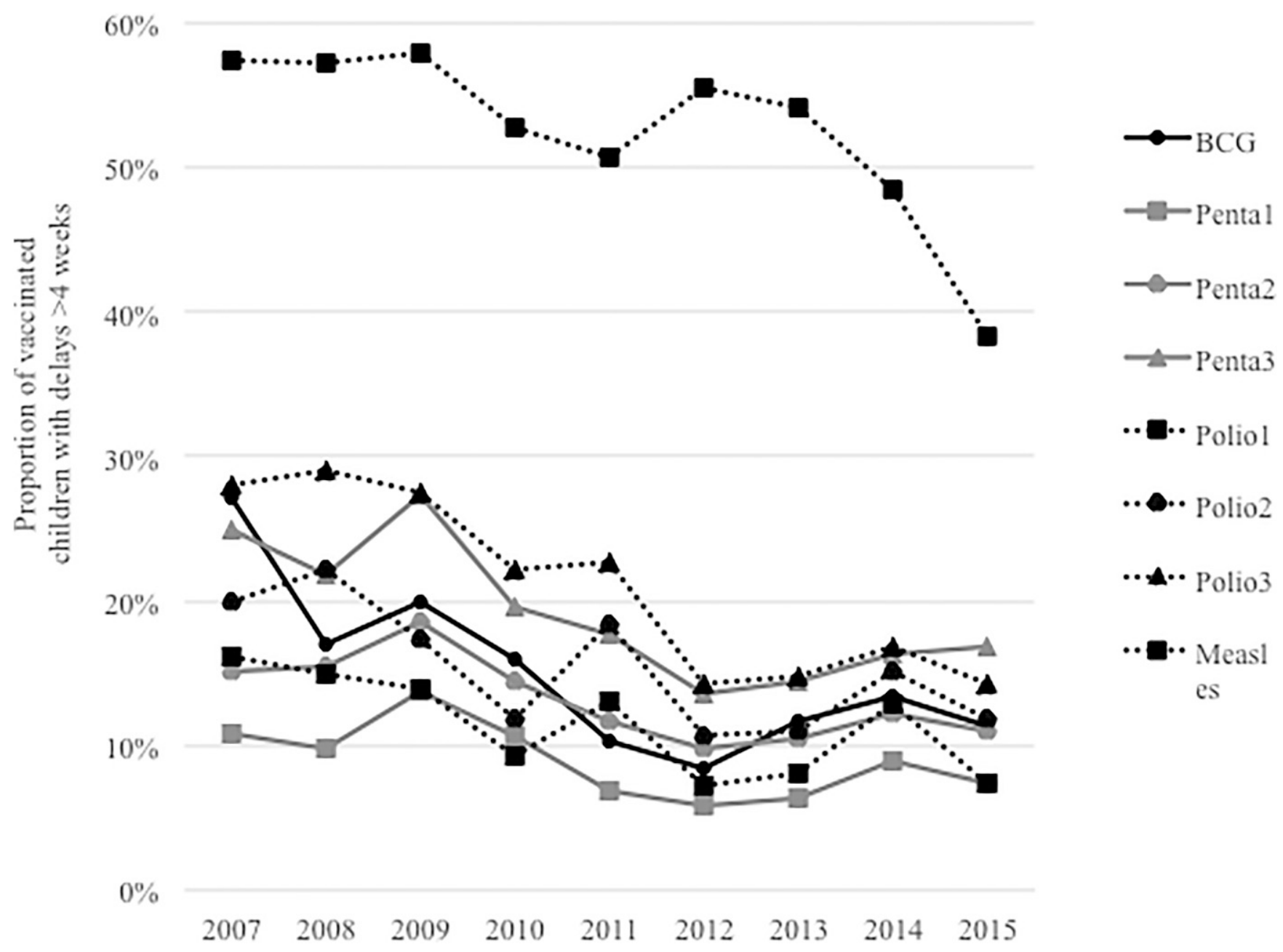

FIGURE 2. Proportion of children 12-23 months delayed in receiving their basic childhood vaccines (BCG, Penta1-3, OPV1-3, and Measles) in Korogocho and Viwandani during 2007-2015, by year.

financially responsible for sustaining the inclusion of PCV as soon as 2027 along with other newly adopted vaccines such as RV and IPV. ${ }^{29}$

The decision to prioritize resources for the continued use of these vaccines in the routine schedule may consider how the new vaccine program fits with the overarching goals of Kenya's immunization program to achieve high coverage (at least $80 \%$ ) in the target age groups for all routine vaccines. ${ }^{30} \mathrm{De}-$ cisions about the introduction of new vaccines into the routine immunization schedule are generally made at the central government level, whereas the responsibility of rollout necessarily resides with the local health authorities. The study of potential systems shocks due to changes in the immunization schedule at more localized levels is important for identifying and addressing any challenges, particularly among target children who are at higher risk for severe illness and premature death associated with their living conditions and circumstances. ${ }^{8,31}$ To our knowledge, this study is the first to show that, following recent new vaccine introduction in a highly vulnerable setting, timeliness of other routine immunization slightly improved or remained neutral to the presence of additional vaccines in the schedule. Without accompanying qualitative understanding of the reception of new vaccines by the communities living in Korogocho and Viwandani, we cannot further substantiate the association, and the comparative prevalence ratios may capture aggregate improvements in vaccination timeliness that initiated well before PCV introduction in 2011. Nonetheless, our findings suggest that the delivery of routine immunization services did not experience disruption or deterioration associated temporally with the incorporation of new vaccines in Korogocho and Viwandani.

When compared with estimated delayed vaccination at the national level, children in Korogocho and Viwandani had substantially higher levels of delayed measles vaccination (54\% overall for the study period compared with an estimated $28 \%$ nationwide in 2014). ${ }^{32}$ Delayed vaccination for other routine doses in Korogocho and Viwandani was lower than the national estimates (BCG: 13.8\% versus 24.3\%; Penta1: $8.1 \%$ versus 10.6\%; Penta2: $12.6 \%$ versus $18.1 \%$; Penta3: $18.2 \%$ versus $24.7 \%$ ), though children belonging to ethnic minorities and lower socioeconomic strata in the two communities had elevated levels of delay in line with the national averages. In contrast, substantial delays in measles vaccination persisted across time and social determinants in both communities. This is particularly concerning in an urban setting where vaccine-preventable disease transmission intensity is likely higher, coupled with the effects of a highly mobile population due to cyclical migration, other food security risks, and 


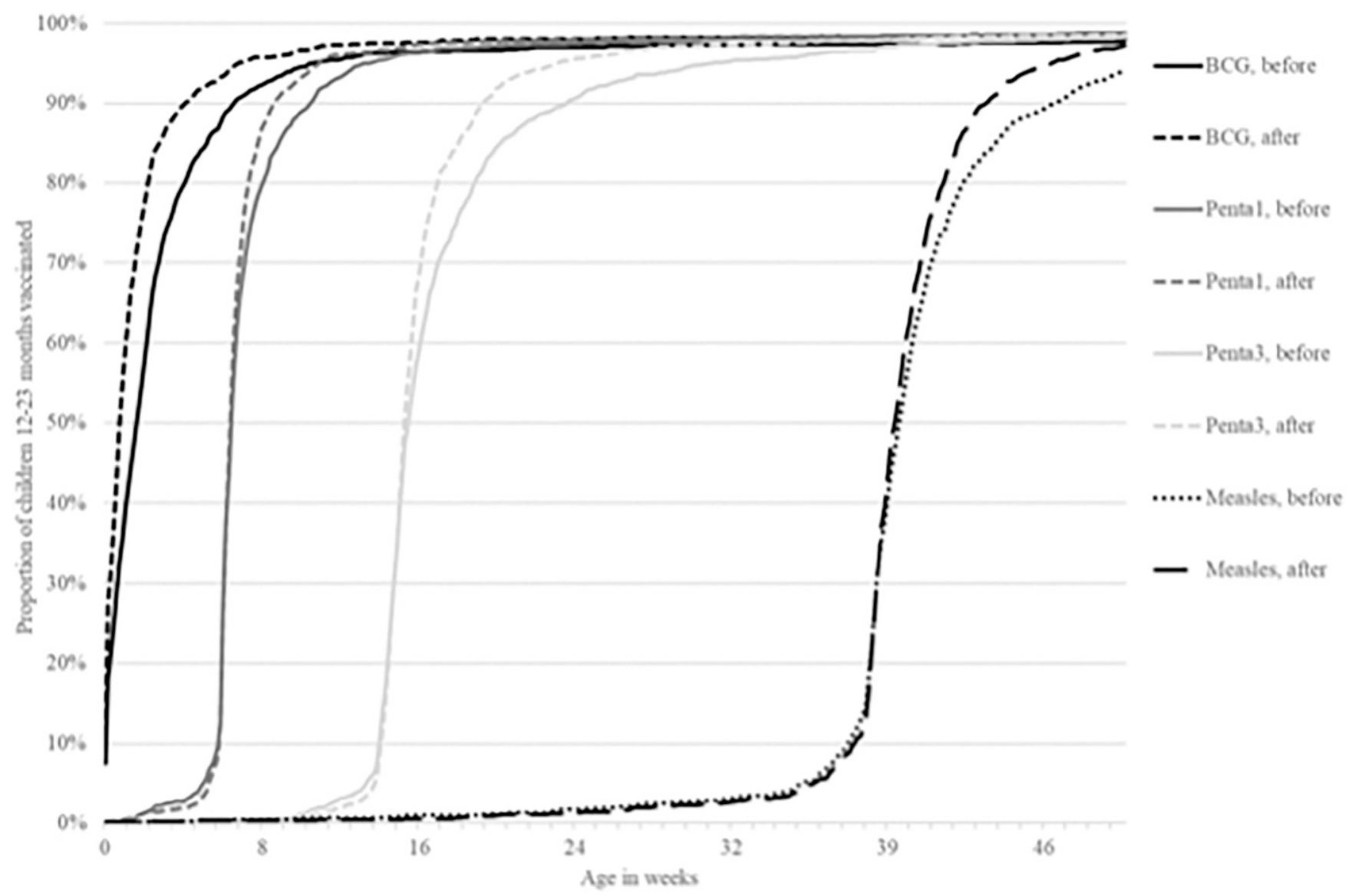

FIGURE 3. Age-specific uptake among vaccinated children 12-23 months registered in the Nairobi Urban Health and Demographic Surveillance System before and after PCV introduction.

sanitation risks, all posing serious challenges for childhood health. ${ }^{31}$

A recent review summarized the variation in definitions and methods used to evaluate vaccination timeliness in LMICs, but it also highlighted the dearth of evidence on age-specific population protection in vulnerable settings, largely due to inadequate readily available data sources. ${ }^{33}$ Our assessment of delayed vaccination over time in Korogocho and Viwandani underscores the extent to which infants are underimmunized for their specific age, contributing to increased risk of vaccine-

TABLE 4

Multivariable associations between time period (2007-2010 vs. 2012-2015) and delays in vaccination for doses at birth, 6 weeks, 14 weeks, and 9 months of age among children 12-23 months registered in the Nairobi Urban Health and Demographic Surveillance System

\begin{tabular}{|c|c|c|c|c|}
\hline & & Preval & $95 \% \mathrm{Cl})$ & \\
\hline & BCG (birth) & Penta1 ( 6 weeks) & Penta3 (14 weeks) & Measles (9 months) \\
\hline Period $\left(\mathrm{ref}^{*}=\right.$ pre [2007-2010]) & & & & \\
\hline Post (2012-2015) & $0.67(0.45-0.99)$ & $0.41(0.24-0.69)$ & $0.59(0.41-0.83)$ & $1.19(0.99-1.42)$ \\
\hline Surveillance site (ref = Korogocho) & & & & \\
\hline Viwandani & $0.89(0.86-1.02)$ & $0.64(0.51-0.80)$ & $0.56(0.48-0.66)$ & $0.93(0.086-0.99)$ \\
\hline Ethnicity (ref = Kikuyu) & & & & \\
\hline Luhya & $2.21(1.76-2.78)$ & $2.50(1.83-3.40)$ & $2.20(1.79-2.69)$ & $1.06(0.97-1.17)$ \\
\hline Luo & $2.04(1.61-2.58)$ & $1.91(1.37-2.67)$ & $1.88(1.51-2.33)$ & $1.00(0.90-1.12)$ \\
\hline Kamba & $1.26(0.96-1.65)$ & $1.30(0.88-1.92)$ & $1.28(0.99-1.66)$ & $1.05(0.95-1.16)$ \\
\hline Kissii & $1.15(0.76-1.74)$ & $1.64(0.95-2.84)$ & $1.35(0.91-2.01)$ & $1.15(1.00-1.32)$ \\
\hline Other & $1.78(1.32-2.39)$ & $2.32(1.58-3.40)$ & $2.01(1.56-2.57)$ & $1.17(1.04-1.31)$ \\
\hline Wealth quintile (ref $=$ poorest) & & & & \\
\hline Poor & $0.86(0.67-1.10)$ & $0.73(0.51-1.04)$ & $0.83(0.66-1.04)$ & $1.02(0.90-1.14)$ \\
\hline Middle & $0.85(0.67-1.08)$ & $0.84(0.60-1.16)$ & $0.93(0.75-1.15)$ & $1.06(0.94-1.18)$ \\
\hline Rich & $0.76(0.60-0.96)$ & $0.80(0.58-1.10)$ & $0.76(0.61-0.94)$ & $0.97(0.86-1.08)$ \\
\hline Richest & $0.68(0.54-0.87)$ & $0.66(0.47-0.91)$ & $0.70(0.57-0.87)$ & $0.94(0.84-1.05)$ \\
\hline Observations & 4,208 & 4,130 & 3,798 & 3,041 \\
\hline
\end{tabular}

BCG $=$ Bacille Calmette-Guerin.

${ }^{*}$ ref $=$ on-time vaccination 
preventable disease outbreaks in these communities. In 2015, fewer than three-quarters of children had completed their recommended immunization schedules for 12 months of age. Another recently published study, with a primary focus on assessing intervenable differences to close the gap between children with complete and incomplete immunization schedules, found that children who had not completed their schedules by 12 months of age were frequently delayed in one or more doses of their vaccination schedule. ${ }^{34}$ With up to $50 \%$ of our sample being delayed in their measles vaccination, there undoubtedly are children who may never complete their immunization schedules and as a result achieve suboptimal protection against the VPDs targeted by the Kenyan immunization program during the vulnerable infancy period. Although on-time vaccination is optimal, recent guidance from $\mathrm{WHO}$ on catch-up vaccination underscores the importance of using every encounter children have with the health system to check their vaccination status and bring them up to date on doses or vaccines that may have been delayed due to access or supply constraints. ${ }^{35}$ This becomes even more important in the context of multi-dose series that have been interrupted, which can lead to suboptimal protection in the long term.

This study has limitations. We were not able to control for several factors that may have a time-specific association with vaccination timeliness, such as anti-vaccine media campaigns, vaccine supply stock-outs, or supplementary immunization activities. Similarly, other vaccines were introduced in addition to PCV during the period evaluated. However, only sufficient time had lapsed since PCV introduction, as opposed to more recently introduced vaccines, to allow for evaluating trends several years before and afterward. Pneumococcal conjugate vaccine is administered without upper-bound age restrictions, even though earlier vaccination is favored. As such, the findings from this analysis cannot necessarily be applied to other new vaccines that may introduce specific barriers or enablers to timely access and demand of the routine schedule. For example, in the presence of upper-limit age restrictions, such was the case initially with $\mathrm{RV}$ introductions, the timeliness of routine immunization might improve, but generally there are no upper age limits in the basic immunization schedule. A second important limitation to our study is the exclusion of children who did not have a vaccination card at the time of interview. The proportion of children who had a card available at the time of interview was not stable over time, ranging from $83 \%$ in 2012 to $44 \%$ in 2010 . If children without vaccination cards discarded or lost them upon completing their schedules on time, then our findings may overestimate the extent of the risk that delayed vaccination poses to these two communities. However, it is more likely that the lack of a vaccination card represents an access barrier to immunization services, and the exclusion of children without cards may underestimate the true prevalence of delays or even nonvaccination. A longitudinal surveillance platform like the NUHDSS is a valuable investment by governments and donors to ensure that health needs are being met in communities that lack coverage of health services and attention from national-scale death registries. However, the setting in which the NUHDSS operates is a challenge to complete follow-up. The average in-migration for children under 5 years of age is $36.9 \%$ annually, whereas the average out-migration is $31.4 \% .^{21}$ This circular migration pattern is an obstacle to obtaining complete immunization records for all births registered in the system, and because of this there may be different levels of representation in our sample from year to year.

\section{CONCLUSION}

In two urban, informal settlements, the introduction of new vaccines was not associated with significantly higher prevalence of delayed vaccination in the routine immunization program. With a large concentration of the urban population residing in informal settlements in Nairobi, on-time and up-todate vaccination is essential for continued progress toward improving childhood health among the most vulnerable. New vaccine introduction should be a used to rejuvenate program goals, increasing coverage to more diseases and improving the uptake and timeliness overall of immunization services.

Received January 3, 2021. Accepted for publication March 22, 2021. Published online May 17, 2021.

Acknowledgments: We thank the Data, Measurement, and Evaluation Unit at the African Population and Health Research Center for their diligent efforts to promote the use of NUHDSS data by researchers external to the organization to evaluate the health and demographic characteristics of populations residing in Korogocho and Viwandani. In particular, we thank Marylene Wamukoya for sharing her wealth of knowledge about the surveillance system. We also thank the NUHDSS participants and data collection team.

Financial support: This work was supported by the African Population and Health Research Center and the Office of Global Public Health at the University of Michigan to conduct research on-site in Nairobi, Kenya.

Authors' addresses: Cara Bess Janusz, School of Public Health, University of Michigan, Ann Arbor, MI, and The Institute of Global Health Equity, University of Michigan, Ann Arbor, MI, E-mail: cjanusz@ umich.edu. Martin K. Mutua, Data Measurements and Evaluation, African Population and Health Research Center, Nairobi, Kenya, E-mail: mkavao@aphrc.org. Abram L. Wagner, University of Michigan, Ann Arbor, MI, E-mail: awag@umich.edu. Matthew L. Boulton, Infectious Disease Division, Michigan Medicine, Ann Arbor, MI, E-mail: mboulton@umich.edu.

\section{REFERENCES}

1. Loharikar A, Dumolard L, Chu S, Hyde T, Goodman T, Mantel C, 2016. Status of new vaccine introduction - worldwide, September 2016. MMWR Morb Mortal Wkly Rep 65: 1136-1141.

2. Gandhi $G, 2015$. Charting the evolution of approaches employed by the Global Alliance for Vaccines and Immunizations (GAVI) to address inequities in access to immunization: a systematic qualitative review of GAVI policies, strategies and resource allocation mechanisms thr. BMC Public Health 15: 1198.

3. Wang SA et al., 2013. New vaccine introductions: assessing the impact and the opportunities for immunization and health systems strengthening. Vaccine 31: 762-773.

4. Mihigo R, Okeibunor J, Anya B, Mkanda P, Zawaira F, 2017. Challenges of immunization in the African Region. Pan Afr Med J 27 (Supp/ 3): 12.

5. World Health Organization, 2020. Geneva, Switzerland: WHO.

6. Phillips DE, Dieleman JL, Lim SS, Shearer J, 2017. Determinants of effective vaccine coverage in low and middle-income countries: a systematic review and interpretive synthesis. BMC Health Serv Res 17: 681.

7. Guerra FA, 2007. Delays in immunization have potentially serious health consequences. Paediatr Drugs 9: 143-148.

8. Mutua MK et al., 2020. Complete and on-time routine childhood immunisation: determinants and association with severe morbidity in urban informal settlements, Nairobi, Kenya. Ann Hum Biol 47: 132-141. 
9. Burchett HED et al., 2014. The impact of introducing new vaccines on the health system: case studies from six low- and middleincome countries. Vaccine 32: 6505-6512.

10. Torres-Rueda $S$ et al., 2015. New pneumococcal conjugate vaccine introductions in four sub-saharan african countries: a cross-country analysis of health systems' impacts. Afr Health Sci 15: 868-877.

11. de Oliveira LH, Trumbo SP, Ruiz Matus C, Sanwogou NJ, Toscano CM, 2016. Pneumococcal conjugate vaccine introduction in Latin America and the Caribbean: progress and lessons learned. Expert Rev Vaccines 15: 1295-1304.

12. de Oliveira LH et al., 2013. Systematic documentation of new vaccine introduction in selected countries of the Latin American Region. Vaccine 31 (Supp/ 3): C114-C122.

13. WHO, 2020.Recommendations for Routine Immunization Schedules, Summary Tables. Geneva, Switzerland: World Health Organization. Available at: https://www.who.int/teams/immunization-vaccinesand-biologicals/policies/who-recommendations-for-routineimmunization-summary-tables. Accessed February 22, 2021.

14. Sambala EZ, Wiyeh AB, Ngcobo N, Machingaidze S, Wiysonge $C S, 2020$. New vaccine introductions in Africa before and during the decade of vaccines - are we making progress? Vaccine 37: 3290-3295.

15. WHO/UNICEF, 2020. Global Summary of Immunization Schedules. WHO/UNICEF Joint Reporting Form. Available at: https://apps. who.int/immunization_monitoring/globalsummary/schedules. Accessed February 22, 2021.

16. Shearer JC, Walker DG, Risko N, Levine OS, 2012. The impact of new vaccine introduction on the coverage of existing vaccines: a cross-national, multivariable analysis. Vaccine 30 : 7582-7587.

17. Ndiritu $\mathrm{M}$ et al., 2006. Immunization coverage and risk factors for failure to immunize within the Expanded Programme on Immunization in Kenya after introduction of new Haemophilus influenzae type $\mathrm{b}$ and hepatitis $\mathrm{b}$ virus antigens. BMC Public Health 6: 1-8.

18. Hull BP, Menzies R, Macartney K, Mclntyre PB, 2013. Impact of the introduction of rotavirus vaccine on the timeliness of other scheduled vaccines: the Australian experience. Vaccine 31: 1964-1969.

19. Zou G et al., 2017. Effects of the introduction of new vaccines in Guinea-Bissau on vaccine coverage, vaccine timeliness, and child survival: an observational study. Vaccine 9: 1-12.

20. Sadoh AE, Nwaneri DU, Ogboghodo BC, Sadoh WE, 2016. Effect of introduction of pentavalent vaccine as replacement for Diphtheria-Tetanus-Pertussis and Hepatitis B vaccines on vaccination uptake in a health facility in Nigeria. Vaccine 34 : 2722-2728.

21. Beguy D et al., 2015. Health and demographic surveillance system profile: the Nairobi Urban Health and Demographic Surveillance System (NUHDSS). Int J Epidemiol 44: 462-471.
22. Beguy D, Bocquier P, Zulu EM, 2010. Circular migration patterns and determinants in Nairobi slum settlements. Demogr Res 23: 549-586.

23. Emina $\mathrm{J}$ et al., 2011. Monitoring of health and demographic outcomes in poor urban settlements: evidence from the Nairobi Urban Health and Demographic Surveillance System. J Urban Health 88: S200-S218.

24. Mutua MK, Kimani-Murage E, Ettarh RR, 2011. Childhood vaccination in informal urban settlements in Nairobi, Kenya: who gets vaccinated? BMC Public Health 11: 6.

25. Beguy $D$ et al., 2015. Health and Demographic Surveillance System Profile: the Nairobi Urban Health and Demographic Surveillance System (NUHDSS). Int J Epidemiol 44: 462-471.

26. Wamukoya M, Kadengye DT, Iddi S, Chikozho C, 2020. The Nairobi Urban Health and Demographic Surveillance of slum dwellers, 2002-2019: value, processes, and challenges. Glob Epidemiol 2: 100024.

27. Olayinka F, Ewald L, Steinglass R, 2017. Beyond new vaccine introduction: the uptake of pneumococcal conjugate vaccine in the African Region. Pan Afr Med J 27 (Supp/ 3): 3.

28. Kallenberg $\mathrm{J}$ et al., 2016. Gavi's transition policy: moving from development assistance to domestic financing of immunization programs. Health Aff (Millwood) 35: 250-258.

29. Ojal $J$ et al., 2019. Sustaining pneumococcal vaccination after transitioning from Gavi support: a modelling and costeffectiveness study in Kenya. Lancet Glob Heal 7: e644-e654.

30. Republic of Kenya Ministry of Public Health and Sanitation, Division of Vaccines and Immunizations, 2013. Comprehensive Multi-Year Plan 2011-2015. Nairobi, Kenya: WHO. Available at: https://www.who.int/immunization/programmes_systems/ financing/countries/cmyp/Kenya_cMYP_doc.pdf. Accessed May 3, 2021.

31. Bocquier P, Beguy D, Zulu EM, Muindi K, Konseiga A, Yé Y, 2011. Do migrant children face greater health hazards in slum settlements? Evidence from Nairobi, Kenya. J Urban Health 88(Supp/ 2): 266-281.

32. Masters NB, Wagner AL, Carlson BF, Boulton ML, 2018. Vaccination timeliness and co-administration among Kenyan children. Vaccine 36: 1353-1360.

33. Masters NB, Wagner AL, Boulton ML, 2019. Vaccination timeliness and delay in low- and middle-income countries: a systematic review of the literature, 2007-2017. Hum Vaccin Immunother 15: 2790-2805.

34. Mutua MK, Kimani-Murage E, Ngomi N, Ravn H, Mwaniki P, Echoka E, 2016. Fully immunized child: coverage, timing and sequencing of routine immunization in an urban poor settlement in Nairobi, Kenya. Trop Med Health 44: 1-12.

35. WHO, 2020. Leave No One Behind: Guidance for Planning and Implementing Catch-up Vaccination. Geneva, Switzerland: World Health Organization. 